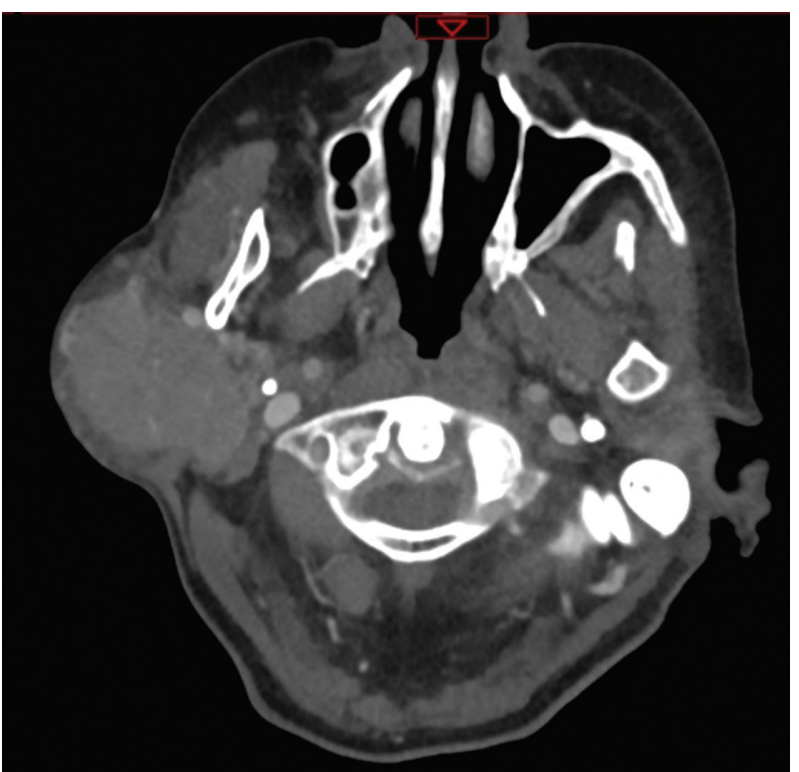

Conclusions: CDS should be considered and craniocervical junction exposed in the context of acute cervical or occipital pain with stiffness and elevated inflammation markers not only in patients previously diagnosed with CPPD, but rather in diverse clinical settings. Particularly, CDS should be recognised as a possible alternative diagnosis in older patients referred with suspicion to giant cell arteritis because of new headache and elevated ESR/CRP. While generally believed to be a rare phenomenon, CDS was seen in 24 patients in 400-bed general hospital within 2 years and is probably widely underdiagnosed.

Disclosure of Interest: None declared

DOI: 10.1136/annrheumdis-2018-eular.3544

\section{FRI0231 STUDY OF URATE TRANSPORTERS IN PRIMARY HYPERURICEMIA AND GOUT}

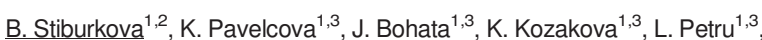
J. Zavada ${ }^{1}$, K. Pavelka ${ }^{1} .{ }^{1}$ Institute of Rheumatology; ${ }^{2}$ Department of Pediatrics and Adolescent Medicine, First Faculty of Medicine, Charles University and General University Hospital in Prague; ${ }^{3}$ Department of Rheumatology, First Faculty of Medicine, Charles University, Prague, Czech Republic

Background: The urate transporters are one of the main genetic determinants of serum uric acid concentrations.

Objectives: In this study we investigated the effects of non-synonymous allelic variants of urate transporters in a cohort of patients with primary gout and/or asymptomatic hyperuricemia

Methods: The cohort consisted of 165 gout patients (151 men, 14 women); 58 hyperuricemic individuals (39 men/19 women); 115 normouricemic controls were used for comparison. Gouty arthritis was diagnosed according to the 1977 preliminary criteria of the American College of Rheumatology. Coding regions of ABCG2, SLC2A9, SLC22A11, SLC22A8, SLC17A3, and SLC17A1 genes were amplified and sequenced directly. To estimate the functions of the identified nonsynonymous allelic variants, we used the protein prediction algorithms.

Results: In ABCG2 gene, we detected nine non-synonymous variants (two common, seven rare including one novel): p.V12M, p.Q141K, p.R147W, p.T153M, p. K360del, p.F373C, p.T434M, p.S476P and p.D620N. The p.Q141K (rs2231142) variant had a significantly higher minor allele frequency $(0.23)$ in the gout patients compared to the European-origin population (0.09) and was significantly more common among gout patients than among normouricemic controls $(\mathrm{OR}=3.15$, $\mathrm{p}<0.0001)$. In addition, patients with non-synonymous $A B C G 2$ allelic variants had an earlier onset of gout ( 41.5 vs. 48 years, $p=0.0478$ ) and a greater likelihood of a familial history of gout $(42 \% \text { vs. } 26 \%, O R=2.02, p=0.043)^{1}$. We identified novel intron variant c. $689+1 \mathrm{G}>\mathrm{A}$ which is associated with two abnormal splicing variants, leading to premature introduction of the stop codon, mislocalized ABCG2 signal on plasma membrane and no urate uptake activity ${ }^{2}$.

In SLC2A9 gene, seven missense variants were identified (six common, one rare): p.A17T, p.G25R, p.T275M, p.D281H, p.V282I, p.R294H, and p.P350L. In
SLC17A3, the analysis revealed common allelic variants p.A100T and p.G279R. Rare non-synonymous variants p.V202M and p.R343L were found in SLC22A11. Only one common missense variant p.T269I was identified in SLC17A1. In SLC22A8 sequencing revealed three rare variants: p.R149C, p.V448I and p. R513G. The precise impact of SLC22A11, SLC22A8, SLC17A3, and SLC17A1 in the context of hyperuricemia and gout in our cohort is unclear and will be further studied include functional characterisation of selected dysfunctional variants. Conclusions: Genetic variants of $A B C G 2$, common and rare, increased the risk of gout and had a significant effect on earlier onset of gout and the presence of a familial gout history. Genotyping the rare variants of $A B C G 2$ along with its common variants is essential for evaluating the individual risk for gout.

\section{REFERENCES:}

[1] Stiburkova B, et al. Functional non-synonymous variants of ABCG2 and gout risk. Rheumatology (Oxford) 2017 Nov 1;56(11):1982-1992.

[2] Stiburkova B, et al. Novel dysfunctional variant in ABCG2 as a cause of severe tophaceous gout: biochemical, molecular genetics and functional analysis. Rheumatology (Oxford) 2016 Jan;55(1):191-4.

Acknowledgements: This study was supported by the grant from the Czech Republic Ministry of Health AZV 15-26693A.

Disclosure of Interest: None declared

DOI: 10.1136/annrheumdis-2018-eular.5311

\section{FRI0232 ULTRASONOGRAPHIC FEATURES OF GOUTY DACTYLITIS OF THE HANDS}

C.A. Guillen-Astete ${ }^{1}$, B. Laso-Jimeno ${ }^{2} .{ }^{1}$ Rheumatology Department, ${ }^{2}$ Ramon y Cajal University Hospital, Madrid, Spain

Background: Dactylitis is a manifestation of gout that can occur on debut or throughout the course of the disease, although it is usually considered a sign of chronicity or a hallmark of long-term disease. Classically, the synovitis or tenosynovitis mediated by the deposit of microcrystals has been interpreted as an inflammatory effect due to proximity. The etiological prevalence based on imaging studies is unknown

Objectives: To determine the prevalence of different ultrasonographic features of dactylitis of the hands in patients with gout.

Methods: A cross-sectional study was conducted based on a registry of ultrasound images of patients with gout and clinical dactylitis either in debut or throughout evolution. The selection of patients followed strictly clinical criteria based on the corresponding medical reports. All images were obtained in a medium-high gamma GE equipment and were obtained by the same operator over three years. Given that no comparisons were planned, no masking of the clinical situation of the patients was made in the eyes of the interpreter. The interpretation of findings was dichotomous in the determination of synovitis, tenosynovitis and enthesopathy according to EULAR definition criteria. The identification of tophi was made according to the definition of Avila Fernandes et al. (doi: 10.1007/s00256-010 $1008-z)$ The overlapping of findings was counted independently at the moment of establishing the prevalence.

Results: We included images of 66 patients diagnosed with gout and with dactylitis of at least one finger at the time of the ultrasound evaluation. The mean age of the patients was 59.2 SD 4.3 years. Sixty-two patients were male. Of the total number of patients, 60 had tenosynovitis of the flexor tendinous apparatus $(90.9 \%)$. Four of these patients also presented tenosynovitis of the tendinous extensor apparatus. No patient presented only extensor tenosynovitis. Enthesopathy was identified in 6 patients $(9.1 \%)$, in no case did enthesopathy occur with power Doppler signal. Synovitis was identified in 43 patients $(65.1 \%)$. Of these, in 13 patients a grade I was registered and in 26 a power Doppler signal was demonstrated. Tophi were identified in 16 patients (242\%).

Conclusions: CONCLUSIONS: This is, as far as we know, the first iconographic study of gouty dactylitis based on ultrasound. According to our results, tenosynovitis of the flexors is the most frequent finding in gouty dactylitis while enthesopathy is rather rare. The presence of significant synovitis is the second most frequent finding while tophi as conditioning agents of synovitis were the least frequent finding. We understand that the knowledge of the echographic characteristics of gouty dactylitis can serve as a clinical guide when making therapeutic decisions in cases were this clinical sign lasts despite the control of other manifestations.

Disclosure of Interest: None declared

DOI: 10.1136/annrheumdis-2018-eular.7070 